\title{
On Convergence Properties of Szasz-Mirakyan-Bernstein Operators of two Variables
}

\author{
Tuncay Tunc \\ Mersin University, Faculty of Science and Letters, Department of Mathematics, Mersin, 33343, Turkey \\ *Corresponding Author: ttunc77@hotmail.com
}

Copyright (C2013 Horizon Research Publishing All rights reserved.

\begin{abstract}
In this study, we have constructed a sequence of new positive linear operators with two variable by using Szasz-Mirakyan and Bernstein Operators, and investigated its approximation properties.
\end{abstract}

Keywords Positive linear operators, Szasz-Mirakyan Operators, Bernstein Polynomials

\section{Introduction}

Let $n \in \mathbb{N}=\{1,2, \ldots\}$ and $f \in C[0,1]$. The $n$th Bernstein polynomial for $f$ is defined by

$$
B_{n} f(x):=\sum_{k=0}^{n} p_{n, k}(x) f\left(\frac{k}{n}\right), \quad x \in[0,1],
$$

where

$$
p_{n, k}(x):=\left(\begin{array}{l}
n \\
k
\end{array}\right) x^{k}(1-x)^{n-k}, \quad k=0,1, \ldots, n .
$$

The Bernstein polynomials are used for important applications in the branches of mathematics, for example, approximation theory, probability theory, number theory, the solution of the integral and differential equations and the others (e.g. [7, 1, 6, 9]).

For $f \in C[0, \infty)$, the Szasz-Mirakyan operators are defined by

$$
S_{n} f(x):=\sum_{k=0}^{\infty} q_{n, k}(x) f\left(\frac{k}{n}\right), x \in[0, \infty), n \in \mathbb{N},
$$

where

$$
q_{n, k}(x):=e^{-n x} \frac{(n x)^{k}}{k !}, \quad k \in \mathbb{N} \cup\{0\} .
$$

Some approximation properties of $S_{n} f$ can be found in works $[3,16,8]$ and references therein.

Now, taking into account the Bernstein polynomials and the Szasz-Mirakyan operators, we introduce some positive linear operators for functions of two variables.
Let $D:=\left\{(x, y) \in \mathbb{R}^{2}: 0 \leq x \leq 1,0 \leq y\right\}$ and $C(D)$ be the set of the real valued continuous functions on $D$. Let us define the operators $L_{n}: C(D) \rightarrow C(D), n \in \mathbb{N}$, as follows: for $f \in C(D)$ and $(x, y) \in D$,

$$
L_{n} f(x, y):=\sum_{m=0}^{\infty} q_{n, m}(y) \sum_{k=0}^{\nu} p_{\nu, k}(x) f\left(\frac{k}{\nu}, \frac{m}{n}\right),
$$

where $q_{n, m}$ and $p_{\nu, k}$ are defined in (4) and (2), respectively, and $\nu:=\nu(m, n)$ is a natural double sequence which is tends to infinity when $m, n \rightarrow \infty$ and $\nu(0,1):=1$. It could be seen easily that the operators $L_{n}$ are linear and positive. They are called as SzaszMirakyan-Bernstein operators.

For $y \in[0, \infty)$ and $f \in C(D)$, let us define the function $f_{y} \in C[0,1]$ by $f_{y}(x):=f(x, y)$. With this notation, the positive linear operators $L_{n}$ given by (5) can be written in the form

$$
L_{n} f(x, y):=\sum_{m=0}^{\infty} q_{n, m}(y) B_{\nu}\left(f_{\frac{m}{n}}\right)(x) .
$$

The function $L_{n} f$ defined by $(6)$ is became the $\nu(0, n)$ th Bernstein polynomial for the function $f_{0}$, on the set $D_{0}:=\left\{(x, y) \in \mathbb{R}^{2}: 0 \leq x \leq 1, y=0\right\}$.

Some positive linear operators for the functions of two variables are introduced and investigated their approximation properties by the authors in $[13,5,11,15,14$, $12,10,4]$.

In this study we investigate some approximation properties of the sequence of positivie linear operators $L_{n}$ defined by (5) in the space of functions which are continuous on compact subsets of $D$, and the order of approximation by means modulus of continuity.

\section{Some Notations and Auxiliary Facts}

1. Let $D:=\left\{(x, y) \in \mathbb{R}^{2}: 0 \leq x \leq 1,0 \leq y\right\}$ and $C(D)$ be the set of the real valued continuous functions on $D$. We denote by $\rho$ a weight function on $D$, that is, a continuous function on $D, \rho(r) \geq 1$ for each $r=$ $(x, y) \in D$ and $\lim _{\|r\| \rightarrow \infty} \rho(r)=\infty$, where $\|\cdot\|$ is the 
Euclidean norm. Let $B_{\rho}(D)$ denote the set of functions defined on $D$ with the condition $|f(r)| \leq M \rho(r)$ for all $r \in D$, where $M$ is a constant depending on $f$, and $C_{\rho}(D):=C(D) \cap B_{\rho}(D) . B_{\rho}(D)$ and $C_{\rho}(D)$ are called weighted function spaces with the norm

$$
\|f\|_{\rho}:=\sup _{(x, y) \in D} \frac{|f(x, y)|}{\rho(x, y)} .
$$

For any nonnegative number $R$, let $D_{R}:=\left\{(x, y) \in \mathbb{R}^{2}\right.$ : $0 \leq x \leq 1,0 \leq y \leq R\}$. Let us denote the space of real valued continuous functions of two variables on $D_{R}$ equipped with the uniform norm:

$$
\|f\|_{C\left(D_{R}\right)}:=\max _{(x, y) \in D_{R}}|f(x, y)|,
$$

by $C\left(D_{R}\right)$.

2. The full modulus of continuity of $f \in C\left(D_{R}\right)$, is denoted by $\omega(f ; \delta), \delta \geq 0$, is defined as follows:

$$
\omega(f ; \delta):=\max \left|f\left(r_{1}\right)-f\left(r_{2}\right)\right|,
$$

where the maximum is taken for all $r_{1}, r_{2} \in D_{R}$ with $\left\|r_{1}-r_{2}\right\| \leq \delta$. The partial modulus of continuity of $f(x, y) \in C\left(D_{R}\right)$ with respect to $x$ is defined by

$$
\omega^{(1)}(f ; \delta):=\max _{0 \leq y \leq R} \max _{\left|x_{1}-x_{2}\right| \leq \delta}\left|f\left(x_{1}, y\right)-f\left(x_{2}, y\right)\right|,
$$

and with respect to $y$ is defined by

$$
\omega^{(2)}(f ; \delta):=\max _{0 \leq x \leq 1} \max _{\left|y_{1}-y_{2}\right| \leq \delta}\left|f\left(x, y_{1}\right)-f\left(x, y_{2}\right)\right| \text {. }
$$

We shall need some well known properties of full and partial modulus of continuity:

$$
\omega(f ; \lambda \delta) \leq(1+\lambda) \omega(f ; \delta)
$$

for any $\lambda \geq 0 . \lim _{\delta \rightarrow 0} \omega(f ; \delta)=0$, when $f$ is uniformly continuous.

For $M>0$ and $0<\alpha \leq 1$, the class of the functions $f \in C(D)$ satisfying the relation

$$
\omega(f ; \delta) \leq M \delta^{\alpha}, \quad \text { for all } \delta \geq 0,
$$

is called a Lipschitz class and denoted by $\operatorname{Lip}_{M}(\alpha)$.

3. Let $x$ be fixed point in $\mathbb{R}$. Let us define the moment functions $E_{i}$ by

$$
E_{i}(s):=(s-x)^{i}, \quad i \in \mathbb{N}_{0} .
$$

Lemma 2.1 (p.14 in [9]) For fixed $x \in[0,1]$, we have

$$
\begin{gathered}
B_{n} E_{0}(x)=1 ; \quad B_{n} E_{1}(x)=0 ; \quad B_{n} E_{2}(x)=\frac{X}{n} \\
B_{n} E_{3}(x)=\frac{(1-2 x) X}{n^{2}} ; \quad B_{n} E_{4}(x)=\frac{3 X^{2}}{n^{2}}+\frac{X-6 X^{2}}{n^{3}},
\end{gathered}
$$
where $X=x(1-x)$.

The following lemma follows by the definition of SzaszMirakyan Operators (3),

Lemma 2.2 For fixed $x \in[0, \infty)$, we have

$$
\begin{gathered}
S_{n} E_{0}(x)=1 ; \quad S_{n} E_{1}(x)=0 ; \quad S_{n} E_{2}(x)=\frac{x}{n} \\
S_{n} E_{3}(x)=\frac{x}{n^{2}} ; \quad S_{n} E_{4}(x)=\frac{3 x^{2}}{n^{2}}+\frac{x}{n^{3}} .
\end{gathered}
$$

\section{Approximation properties of $L_{n}$ on $C\left(D_{R}\right)$}

Let $L_{n}$ be the positive linear operators defined by (5) with the condition $\nu=O(m), m \rightarrow \infty$, that is, there exists natural sequences $\alpha_{n}$ and $\beta_{n}$ such that $\alpha_{n} \leq \nu / m \leq \beta_{n}$. In this section we give some classical approximation properties of the operators $L_{n}$. Let $e_{j}, j=0,1,2,3$ be the test functions defined by

$$
\begin{gathered}
e_{0}(x, y):=1, e_{1}(x, y):=x, \\
e_{2}(x, y):=y, e_{3}(x, y):=x^{2}+y^{2} .
\end{gathered}
$$

By simple calculations, we get the following lemma.

Lemma 3.1 For each $n \in \mathbb{N}$, we have

$$
\begin{aligned}
& L_{n} e_{j}(x, y)=e_{j}(x, y), \quad \text { for } j=0,1,2 \\
& L_{n} e_{3}(x, y)=e_{3}(x, y)+\frac{y}{n}+X \sum_{m=0}^{\infty} \frac{e^{-n y}(n y)^{m}}{m ! \nu} .
\end{aligned}
$$

where $X=x(1-x)$.

The following theorem gives the Baskakov-type theorem (see [2]) to get uniform approximation to the functions in $C\left(D_{R}\right)$ satisfying some additional conditions by the sequence of the positive linear operators $L_{n}$.

Theorem 3.2 For the sequence of positive linear operators $L_{n}$, the convergence

$$
\left\|L_{n} e_{j}-e_{j}\right\|_{C\left(D_{R}\right)} \rightarrow 0, n \rightarrow \infty, j=0,1,2,3
$$

implies that

$$
\left\|L_{n} f-f\right\|_{C\left(D_{R}\right)} \rightarrow 0, \quad n \rightarrow \infty,
$$

for all $f \in C_{\rho}(D)$ with $\rho(x, y)=2+y^{2}$.

Proof. Let $f \in C_{\rho}(D)$ with $\rho(x, y)=2+y^{2}$ and $(x, y) \in D_{R}$. By the continuity of $f$ at the point $(x, y)$, for any positive number $\epsilon$, there exists a number $\delta>0$ such that for all $(s, t) \in D$ satisfying $\sqrt{(s-x)^{2}+(t-y)^{2}}<\delta$, the inequality $|f(s, t)-f(x, y)|<\epsilon$ holds. Since $f \in B_{\rho}(D)$, there exists a number $M>0$, such that $|f(x, y)| \leq M\left(2+y^{2}\right)$ for $(x, y) \in D$. Hence, for $(s, t) \in D$ satisfying $\sqrt{(s-x)^{2}+(t-y)^{2}} \geq \delta$, we have

$$
\begin{aligned}
|f(s, t)-f(x, y)| & \leq M\left(4+t^{2}+y^{2}\right) \\
& \leq M_{1} \frac{(s-x)^{2}+(t-y)^{2}}{\delta^{2}}
\end{aligned}
$$

where $M_{1}>0$ is a constant depending on $f$ and $R$. Therefore, we obtain that for $(s, t) \in D$

$$
|f(s, t)-f(x, y)|<\epsilon+M_{1} \frac{(s-x)^{2}+(t-y)^{2}}{\delta^{2}} .
$$

Applying the operators $L_{n}$ to the last inequality, we get

$$
\begin{gathered}
\left|L_{n} f(x, y)-f(x, y)\right| \leq L_{n}(|f(., .)-f(x, y)|)(x, y) \\
+\|f\|_{C\left(D_{R}\right)}\left|L_{n} e_{0}(x, y)-e_{0}(x, y)\right|
\end{gathered}
$$




$$
\begin{aligned}
\leq & \epsilon L_{n} e_{0}(x, y)+\frac{M_{1}}{\delta^{2}}\left(L_{n} e_{3}(x, y)-2 x L_{n} e_{1}(x, y)\right. \\
& \left.-2 y L_{n}\left(e_{2}\right)(x, y)+\left(x^{2}+y^{2}\right) L_{n} e_{0}(x, y)\right) \\
& +\|f\|_{C\left(D_{R}\right)}\left|L_{n} e_{0}(x, y)-e_{0}(x, y)\right|
\end{aligned}
$$

By using the assumptions (12), the desired assertion (13) is proved.

Theorem 3.3 For any $f \in C_{\rho}(D)$ with $\rho(x, y)=2+$ $y^{2}$, the sequence $\left\{L_{n}(f)\right\}$ converges uniformly to $f$ on arbitrary compact subset $G$ of $D$.

Proof. Without loss of generality, we can assume that $G=D_{R}$ for some $R \geq 0$. Since the sequence of functions $\left\{g_{n}\right\}$, where $g_{n}(x, y):=\frac{y}{n}+x(1-x) \sum_{m=0}^{\infty} \frac{e^{-n y}(n y)^{m}}{m ! \nu}$, converges uniformly to zero on $D_{R}$, the proof is clear by Lemma 3.1 and Theorem 3.2.

The rates of convergence of the sequence $\left\{L_{n} f\right\}$ to $f$ by means of full and partial modulus of continuity are given in the following theorem.

Theorem 3.4 For $f \in C\left(D_{R}\right)$, we have

$$
\begin{gathered}
\left\|L_{n} f-f\right\|_{C\left(D_{R}\right)} \leq \frac{3}{2} \sum_{i=1}^{2} \omega^{(i)}\left(f ; \delta_{n, i}\right), \\
\left\|L_{n} f-f\right\|_{C\left(D_{R}\right)} \leq \frac{3}{2} \omega\left(f ; \delta_{n}\right),
\end{gathered}
$$

where $\delta_{n, 1}=\frac{1}{\sqrt{n}}, \delta_{n, 2}=\sqrt{\frac{4 R}{n}}$ and $\delta_{n}=\sqrt{\frac{4 R+1}{n}}$.

Proof. For $(x, y) \in D_{R}$,

$$
\begin{gathered}
\left|L_{n} f(x, y)-f(x, y)\right| \leq \\
\sum_{m=0}^{\infty} q_{n, m}(y) \sum_{k=0}^{\nu} p_{\nu, k}(x)\left|f\left(\frac{k}{\nu}, \frac{m}{n}\right)-f(x, y)\right| .
\end{gathered}
$$

First, using the inequality

$$
\begin{gathered}
\left|f\left(\frac{k}{\nu}, \frac{m}{n}\right)-f(x, y)\right| \leq\left(1+\frac{\left|\frac{m}{n}-y\right|}{\delta_{n, 2}}\right) \omega^{(2)}\left(f ; \delta_{n, 2}\right) \\
+\left(1+\frac{1}{\delta_{n, 1}}\left|\frac{k}{\nu}-x\right|\right) \omega^{(1)}\left(f ; \delta_{n, 1}\right)
\end{gathered}
$$

which is obtained from (10), then applying the CauchySchwartz inequality, finally, using Lemma 3.1, the inequality (16) gives the inequality (14). By similar arguments with the inequality

$$
\begin{gathered}
\left|f\left(\frac{k}{\nu}, \frac{m}{n}\right)-f(x, y)\right| \leq \\
\left(1+\frac{1}{\delta_{n}} \sqrt{\left(\frac{k}{\nu}-x\right)^{2}+\left(\frac{m}{n}-y\right)^{2}}\right) \omega\left(f ; \delta_{n}\right),
\end{gathered}
$$

we get the inequality (15).

Corollary 3.5 Let $f \in C\left(D_{R}\right)$. If $f \in \operatorname{Lip}_{M}(\alpha), 0<$ $\alpha \leq 1$, then

$$
\left\|L_{n}(f)-f\right\|_{C\left(D_{R}\right)} \leq \frac{3 M}{2} \delta_{n}^{\alpha}
$$

holds, where $\delta_{n}$ is defined in Theorem 3.4.

\subsection{Voronovskaya-Type Theorem}

From now on we make the assumption: $\nu(m, n)=$ $(m+1) n$, for all $m+1, n \in \mathbb{N}$. Let $(x, y)$ be a fixed point in $\mathbb{R}^{2}$. Let us define the moment functions $E_{i, j}$ by

$$
E_{i, j}(s, t):=(s-x)^{i}(t-y)^{j}, \quad i, j \in \mathbb{N}_{0}
$$

Using Lemma 2.1 and Lemma 2.2, we get the following lemma.

Lemma 3.6 Let $(x, y) \in D \backslash D_{0}$. For each $n \in \mathbb{N}$, we have

$$
\begin{aligned}
L_{n} E_{0, j}(x, y) & =S_{n} E_{j}(y) \\
L_{n} E_{1, j}(x, y) & =0, \quad \text { for } j \in \mathbb{N}_{0} ; \\
L_{n} E_{2,0}(x, y) & =\frac{X}{n^{2} y} H_{0}(n y) \\
L_{n} E_{2,2}(x, y) & =\frac{X}{n^{3} y}\left(y+\frac{1}{n}-\frac{(1+n y)^{2}}{n e^{n y}}\right) \\
L_{n}\left(E_{4,0}\right)(x, y) & =\frac{3 X^{2}}{n^{3} y} H_{1}(n y)+\frac{X-6 X^{2}}{n^{4} y} H_{2}(n y),
\end{aligned}
$$

where $X=x(1-x)$ and

$$
H_{s}(x)=e^{-x} \sum_{m=1}^{\infty} \frac{x^{m}}{m ! m^{s}}, \quad s \in \mathbb{N}_{0} .
$$

By simple calculations, it can be obtained that

$$
H_{s}(x)=\frac{c_{s}}{x^{s}}\left(1-e^{x} \sum_{m=0}^{s} \frac{x^{m}}{m !}\right)
$$

where $1 \leq c_{s} \leq(s+1)$ !, and hence for fixed $x \in(0, \infty)$, $\lim _{x \rightarrow \infty} x^{s} H_{s}(x)=c_{s}$.

Corollary 3.7 If $(x, y) \in D_{0}$, then

$$
\lim _{n \rightarrow \infty} n^{2} L_{n}\left(E_{4,0}+E_{0,4}\right)(x, y)=3 x(1-x)
$$

and, if $(x, y) \in D \backslash D_{0}$, then

$$
\lim _{n \rightarrow \infty} n^{2} L_{n}\left(E_{4,0}+E_{0,4}\right)(x, y)=3 y^{2}
$$

We shall denote by $f_{x}^{\prime}, f_{x x}^{\prime \prime}$ the partial derivatives of $f$. Let us denote the space of functions have continuous partial derivatives up to order 2 on $D$, by $C^{2}(D)$.

Theorem 3.8 Suppose that $f \in C^{2}(D)$. Then, for $(x, y) \in D_{0}$, we have

$$
\lim _{n \rightarrow \infty} n\left(L_{n} f(x, y)-f(x, y)\right)=\frac{x(1-x)}{2} f_{x x}^{\prime \prime}(x, y)
$$

and for $(x, y) \in D \backslash D_{0}$, we have

$$
\left.\lim _{n \rightarrow \infty} n\left(L_{n} f(x, y)-f(x, y)\right)=\frac{y}{2} f_{y y}^{\prime \prime}(x, y)\right) .
$$

Proof. Let $(x, y)$ be a fixed point in $D$. By the Taylor formula for $f \in C^{2}(D)$, we have

$$
\begin{gathered}
f(s, t)=f(x, y)+f_{x}^{\prime}(x, y)(s-x)+f_{y}^{\prime}(x, y)(t-y) \\
+\frac{1}{2} f_{x x}^{\prime \prime}(x, y)(s-x)^{2}+f_{x y}^{\prime \prime}(x, y)(s-x)(t-y)
\end{gathered}
$$


$+\frac{1}{2} f_{y y}^{\prime \prime}(x, y)(t-y)^{2}+\psi(s, t, x, y) \sqrt{(s-x)^{4}+(t-y)^{4}}$, for $(s, t) \in D$, where $\psi(\cdot, \cdot, x, y)=\psi \in C(D)$ and $\psi(x, y)=0$. Thus, we get

$$
\begin{gathered}
L_{n} f(x, y)=f(x, y)+f_{x}^{\prime}(x, y) L_{n} E_{1,0}(x, y) \\
+f_{y}^{\prime}(x, y) L_{n} E_{0,1}(x, y)+\frac{1}{2} f_{x x}^{\prime \prime}(x, y) L_{n} E_{2,0}(x, y) \\
+f_{x y}^{\prime \prime}(x, y) L_{n} E_{1,1}(x, y)+\frac{1}{2} f_{y y}^{\prime \prime}(x, y) L_{n} E_{0,2}(x, y) \\
+L_{n}\left(\psi \cdot \sqrt{E_{4,0}+E_{0,4}}\right)(x, y)
\end{gathered}
$$

Applying the Cauchy-Schwartz inequality, we have

$$
\begin{gathered}
\left|L_{n}\left(\psi \cdot \sqrt{E_{4,0}+E_{0,4}}\right)(x, y)\right| \leq \\
\left(L_{n}\left(\psi^{2}\right)(x, y)\right)^{1 / 2}\left(L_{n}\left(E_{4,0}+E_{0,4}\right)(x, y)\right)^{1 / 2} .
\end{gathered}
$$

Theorem 3.4 implies that

$$
\left.\lim _{n \rightarrow \infty}\left(L_{n}\left(\psi^{2}\right)(x, y)\right)\right)=\psi^{2}(x, y)=0
$$

Using (21) and Corollary 3.7, we obtain from (20)

$$
\lim _{n \rightarrow \infty} n L_{n}\left(\psi \cdot \sqrt{E_{4,0}+E_{0,4}}\right)(x, y)=0 .
$$

Using (22) and Lemma 3.6, we derive (17) and (18) from (19). Thus the proof is completed.

\subsection{Partial Derivatives}

Let us assume that $\nu=n(m+1)$ in the operators $L_{n}$ defined by (5).

Theorem 3.9 Let $f \in C^{1}(D)$. Then for every $(x, y) \in$ D

$$
\lim _{n \rightarrow \infty} \frac{\partial}{\partial y} L_{n} f(x, y)=f_{y}^{\prime}(x, y) .
$$

Proof Let $(x, y)$ be a fixed point in $D$. From (5) it follwos that

$$
\frac{\partial}{\partial y} L_{n} f(x, y)=\frac{n}{y} L_{n} g(x, y)-n L_{n} f(x, y)
$$

where $g(x, y):=y f(x, y)$. By the Taylor formula for $f \in C^{1}(D)$, we have

$$
\begin{gathered}
f(s, t)=f(x, y)+f_{x}^{\prime}(x, y)(s-x)+f_{y}^{\prime}(x, y)(t-y) \\
+\psi(s, t) \sqrt{(s-x)^{2}+(t-y)^{2}}
\end{gathered}
$$

for $(s, t) \in D$, where $\lim _{(s, t) \rightarrow(x, y)} \psi(s, t)=\psi(x, y)=0$. Thus, we get

$$
L_{n} f(x, y)=f(x, y)+L_{n}\left(\psi \sqrt{E_{2,0}+E_{0,2}}\right)(x, y)
$$

and

$$
\begin{aligned}
& L_{n} g(x, y)=y f(x, y)+\frac{y}{n} f_{y}^{\prime}(x, y) \\
& +L_{n}\left(\psi E_{0,1} \sqrt{E_{2,0}+E_{0,2}}\right)(x, y) \\
& +y L_{n}\left(\psi \sqrt{E_{2,0}+E_{0,2}}\right)(x, y) .
\end{aligned}
$$

Using these equations we can rewrite (23) as

$$
\begin{gathered}
\frac{\partial}{\partial y} L_{n} f(x, y)= \\
f_{y}^{\prime}(x, y)+\frac{n}{y} L_{n}\left(\psi E_{0,1} \sqrt{E_{2,0}+E_{0,2}}\right)(x, y)
\end{gathered}
$$

The proof is completed by showing that the last term of the right hand of (24) tends to zero when $n \rightarrow \infty$. By Cauchy-Schwartz Inequality it follows that

$$
\begin{gathered}
\left|L_{n}\left(\psi E_{0,1} \sqrt{E_{2,0}+E_{0,2}}\right)(x, y)\right| \leq \\
\left(L_{n}\left(\psi^{2}\right)(x, y)\right)^{1 / 2}\left(L_{n}\left(E_{2,2}+E_{0,4}\right)(x, y)\right)^{1 / 2} .
\end{gathered}
$$

Since by Lemma 3.6

$$
\lim _{n \rightarrow \infty} n^{2} L_{n}\left(E_{2,2}+E_{0,4}\right)(x, y)=3 y^{2}
$$

and by (15)

$$
\lim _{n \rightarrow \infty} L_{n}\left(\psi^{2}\right)(x, y)=\psi^{2}(x, y)=0,
$$

then we have desired result.

Remark 3.10 The method of the proof of the Theorem 3.9 does not work to prove of convergence of partial derivative of $L_{n} f$ with respect to $x$.

\section{REFERENCES}

[1] G. J. Babu, A. Canty, Y. Chaubey. Application of Bernstein polynomials for smooth estimation of a distribution and density function, J. Stat.Plann. Inference Vol. 105 No.2 377-392.

[2] V. V. Baskakov. On a construction of converging sequences of linear positive operators, Studies od modern problems of Constructive Theory of Functions (1961) 314-318.

[3] M. Becker, Global approximation theorems for SzaszMirakyan and Baskakov operators in polynomial weight spaces, Indiana Univ. Math. J. Vol.27 No.1, 127-142.

[4] I. Buyukyazici, E. Ibikli. The approximation porperties of generalized Bernstein Polynomials of two variables, Appl. Math. Comput. Vol.156 (2004) 367-380.

[5] A. D. Gadjiev. Positive linear operators in weighted spaces of function of several variables, Izv.Akad. Nauk Azerbaidjan, SSR Ser.Fiz-Tek. Mat. Nauk Vol.1 No.4, $32-37$.

[6] C. W. Groetsch, J. T. King. The Bernstein Polynomials and finite differences, Math. Mag. Vol.46 (1973), 280-282.

[7] C. Heitzinger, S. Selberherr. Optimization for TCAD purposes using Bernstein polynomials, Proceeding SISPAD 2001 Conf. 420-423, 2002. 
[8] T. Hermann. On the Szasz-Mirakyan operator, Acta Math. Acad. Sci. Hun. Vol.32 No.1-2, 163-173.

[9] G. G. Lorentz. Bernstein Polynomials, Toronto, 1953.

[10] L. Rempulska, S Graczyk. On Generalized SzaszMirakyan Operators of functions of two variables, Math. Slovaca. Vol.62 No.1, 87-98.

[11] D. D. Stancu. A new class of uniform approximating polynomial operators in two and several variables, Prooc. of Conf. on the Constr. Theory of Func. (Approximation Theory, Budapest,1969), 443-455, 1972.

[12] F. Tasdelen, A. Olgun, G. B. Tunca. Approximation of functions of two variables by certain linear positive operators, Proc. Indian Acad. Sci. Vol.117 No.3, 387-399.
[13] V. I. Volkov. On the convergence of sequences of linear positive operators in the space of two variables, Dokl. Akad. Nauk. SSSR (N.S.) Vol. 115(1957), 17-19.

[14] Z. Walczak. Approximation properties of certain linear positive operators in exponential weighted spaces of functions of two variables, Acta Univ. Palacki. Olomuc., Fac. rer. nat., Mathematica Vol.42(2003) 131-138.

[15] Z. Walczak. Approximation of functions of two variables by some linear positive operators, Acta Math. Univ. Comenianae, LXXIV(1) 37-48, 2005.

[16] D. X. Zhou. Weighted approximation by SzaszMirakyan operators, J. Approx. Th. Vol.76 No.3, 393402. 